Cuadernos de Filología Italiana

ISSN: $1133-9527$

http://dx.doi.org/10.5209/CFIT.57224

\title{
Frasi nominali in un corpus di verbali di interventi chirurgici
}

\author{
Elisa D’Argenio'; Cesarina Vecchia ${ }^{2}$
}

Ricevuto 13 novembre 2017: / Modificato: 03 marzo 2018 / Accettato: 22 maggio 2018

Riassunto. Lo studio si propone di illustrare alcune frasi nominali tratte da un corpus di descrizioni di interventi chirurgici. In tali documenti è ampiamente confermata la preferenza tipica dei testi delle prassi mediche per la predicazione nominale in luogo di quella verbale. Il corpus infatti presenta numerose frasi nominali e, con particolare frequenza, costruzioni formate da un nome d'azione con argomento introdotto da preposizione. Accanto a costruzioni di questo tipo compaiono strutture non prototipiche in cui sono giustapposti un nome d'azione e un nome concreto; in questi stessi contesti ricorre inoltre una struttura in cui si attesta il solo nome concreto. L'analisi mostrerà come determinate caratteristiche testuali delle descrizioni di interventi chirurgici possano contribuire all'emergere di frasi nominali con struttura non prototipica attraverso fenomeni di ellissi grammaticale e contestuale.

Parole chiave: linguaggio medico; frase nominale; nominalizzazione; ellissi.

\section{[en] Noun Phrases in a corpus of medical reports}

Abstract. The aim of this study is to describe some Noun Phrases collected in a corpus of medical reports. As is well known, this kind of texts is characterised by a wide use of Noun Phrases. In particular, the analysis focuses on action noun constructions formed by an action noun and its argument introduced by a preposition. In addition to this, non-prototypical structures also occur in the corpus. One case consists of an action noun which is juxtaposed to a concrete noun. Another case consists of a structure without the action noun in which only the concrete noun is attested. This paper will thus attempt to demonstrate the role played by certain textual features of medical reports on the emerging of nonprototypical Noun Phrases as a result of a variety of ellipse phenomena.

Key words: medical language; Noun Phrase; nominalization; ellipse.

Sommario: 1. Introduzione 2. Il corpus 3. Caratteristiche linguistiche del corpus 4. Tipologie di frasi nominali nel corpus 5. Costruzioni nominali con nomi d'azione 6. Conclusioni.

Come citare: D’Argenio, Elisa / Vecchia, Cesarina (2018) «Frasi nominali in un corpus di verbali di interventi chirurgici», Cuadernos de Filología Italiana, 25, pp. 37-54.

1 Università degli Studi di Verona. Dipartimento di Culture e Civiltà, viale dell’Università, 4 - 37129 - Verona. elisa.dargenio@gmail.com.

2 Università degli Studi di Napoli Federico II. Dipartimento di Studi Umanistici, via Porta di Massa, 1 - 80133 Napoli. cesarina.vecchia@unina.it 


\section{Introduzione ${ }^{3}$}

In questo lavoro si mostrerà come determinate caratteristiche testuali dei verbali di interventi chirurgici possano contribuire all'emergere di frasi nominali con struttura non prototipica risultato di ellissi grammaticale e contestuale. Il fenomeno di ellissi si estende in questi testi fino ad erodere sul piano sintattico i costituenti fondamentali della frase nominale, senza tuttavia mai comprometterne la comprensibilità.

I verbali di interventi chirurgici rappresentano una tipologia testuale con proprietà non del tutto assimilabili a quelle di altri tipi di testi specialistici medici maggiormente indagati in bibliografia: testi teorico-speculativi (manuali, articoli scientifici, trattati), testi destinati al largo pubblico (ricettari e foglietti illustrativi), testi delle prassi rivolti agli operatori di settore (referti strumentali, cartelle cliniche $)^{4}$. I verbali di interventi chirurgici rientrano in quest'ultima categoria ma, sebbene condividano con i referti strumentali alcune caratteristiche linguistiche e testuali, presentano una fisionomia propria e una struttura ben definita, meno dipendente dal singolo caso clinico e fortemente vincolata alle specificità dell'intervento chirurgico descritto.

\section{Il corpus}

Il corpus in esame è estratto da 4.035 verbali di interventi chirurgici ${ }^{5}$. Il verbale rappresenta un rapporto dettagliato dell'operazione chirurgica redatto immediatamente dopo l'intervento nell'apposito registro di camera operatoria e successivamente inserito nella cartella clinica del paziente. Il verbale operatorio assolve alla funzione giuridica di certificare l'attività sanitaria svolta e di documentare le modalità di esecuzione degli interventi chirurgici e delle altre procedure invasive eseguite. Il rapporto contiene gli identificativi numerici del paziente e degli operatori, la tempistica dell'intervento, la diagnosi iniziale e quella finale, l'indicazione della tipologia di anestesia adoperata, la denominazione del tipo di intervento chirurgico e una descrizione sufficientemente dettagliata della procedura chirurgica eseguita. Nel verbale a ciascuna di queste informazioni è riservato uno spazio specifico all'interno di una struttura modulare a campi predefiniti. I testi contenuti nel campo "Descrizione", di cui si mostrano di seguito alcuni esempi, costituiscono il corpus di riferimento dell'analisi:

3 La ricerca è stata condotta presso il Dipartimento di Ingegneria Elettrica e delle Tecnologie dell'Informazione, Università degli Studi di Napoli Federico II, nell'ambito del progetto Nuovi Paradigmi e Tecnologie per la Collective Knowledge nell'e-society, Responsabile scientifico Prof. Antonino Mazzeo. Il progetto è stato finanziato con fondi POR Campania FSE 2007/2013, asse IV e asse V, Sviluppo di reti di eccellenza tra Università - Centri di ricerca - Imprese. Sebbene il contributo sia frutto del lavoro congiunto delle due autrici, i paragrafi 1, 4 e 6 sono da attribuire ad entrambe, i paragrafi 2, 3, 5.1 .3 e 5.1.4 a Elisa D’Argenio, i paragrafi 5, 5.1, 5.1.1 e 5.1.2 a Cesarina Vecchia.

4 A riguardo si vedano, tra gli altri, Cortelazzo (1994: 27-44) e Serianni (1989: 381-420; 2003: 79-106, 2005).

5 Si tratta di verbali provenienti da un'unica struttura ospedaliera che per evidenti ragioni di privacy non sarà resa nota. I verbali sono relativi ad interventi di chirurgia generale e sono stati redatti nell'arco di 5 anni da parte di 16 diversi chirurghi. 
Esempi dei campi "TipoIntervento" e "Descrizione".

\begin{tabular}{|l|l|}
\hline \multicolumn{1}{|c|}{ TipoIntervento } & \multicolumn{1}{c|}{ Descrizione } \\
\hline colecistectomia & $\begin{array}{l}\text { induzione del pneumoperitoneo con ago di Veress. Posizionamento dei trocars } \\
\text { ai soliti punti di repere con 5 mm. All'ombelico. Colecisti in preda a flogosi } \\
\text { acuta con aderenze tra infundibolo e duodeno. Lisi delle aderenze, preparazione } \\
\text { degli elementi del peduncolo e sezione tra clips degli stessi. Colecistectomia } \\
\text { ed estrazione della colecisti con endobag, toilette del cavo, drenaggio in loggia } \\
\text { sottoepatica estrazione sotto visione dei trocars, sutura delle breccie chirurgiche. }\end{array}$ \\
\hline $\begin{array}{l}\text { alloplastica sec. } \\
\text { Liechtenstein }\end{array}$ & $\begin{array}{l}\text { incisione inguinale a ds. aperto il canale inguinale si apprezza un grosso } \\
\text { sacco erniario da ernia obliqua esterna. resezione ed affondamento del sacco, } \\
\text { posizionamento di rete in polipropilene con tecnica di liechtenstein, controllo } \\
\text { dell'emostasi, sutura della fascia e della parete a strati. }\end{array}$ \\
\hline
\end{tabular}

I testi in esame sono organizzati secondo una successione di sequenze testuali corrispondenti ai tempi delle prassi chirurgiche e presentano dunque una struttura ordinata in segmenti che possono essere considerati come unità informative minime rispetto all'intera descrizione dell'intervento. Tali unità saranno qui definite "sezioni informative" ". L'individuazione delle sezioni informative ha permesso di sviluppare un modello di riferimento che rappresentasse la specifica sequenza dei tempi e delle azioni chirurgiche che caratterizza ciascuna tipologia di intervento presente nel corpus. Tale modello, la cui validità è stata successivamente verificata attraverso la consultazione di bibliografia medica ${ }^{7}$, costituisce la struttura dei testi indagati.

Di seguito si mostrano le strutture in sezioni informative delle descrizioni dei due interventi maggiormente attestati nel corpus, la colecistectomia video-laparoscopica (VLS) e la chirurgia dell'ernia inguinale:

Struttura in sezioni informative dell'intervento di "colecistectomia VLS"

\section{Colecistectomia VLS}

- Induzione del pneumoperitoneo

- Introduzione dei trocars

- Lisi delle aderenze

- Isolamento degli elementi del peduncolo

- Legatura degli elementi del peduncolo

- Sezione degli elementi del peduncolo

- Colecistectomia VLS

- Controllo dell'emostasi

- Toilette del cavo peritoneale

- Posizionamento del drenaggio

- Estrazione dei trocars

- Sutura delle incisioni chirurgiche
Struttura in sezioni informative dell'intervento di "chirurgia dell'ernia inguinale"

\section{Chirurgia dell'ernia inguinale}

- Incisione inguinale

- Apertura del canale inguinale

- Preparazione del funicolo

- Isolamento del sacco erniario

- Sezione del sacco erniario

- Affondamento del sacco erniario

- Rinforzo della parete posteriore

- Rinforzo dell'anello inguinale interno

- Alloplastica erniaria

- Controllo dell'emostasi

- Toilette

- Posizionamento del drenaggio

- Sutura delle incisioni chirurgiche

6 Si precisa che la terminologia "sezione informativa" proposta non coincide con il più complesso concetto di "unità informativa". Con "sezione informativa" si intende qui un segmento di testo che può essere interpretato come unità tematica minima e non ulteriormente analizzabile rispetto al più articolato quadro informativo di un testo considerato nella sua interezza.

7 In particolare sono stati consultati Kremer (1990-1999), Paletto (1996-2006) e Vella (2008). 
Come si vedrà, la definizione di un modello di riferimento si è rivelata utile in fase di analisi per valutare la variazione e le diverse realizzazioni delle costruzioni nominali oggetto di questo studio. Tali forme, infatti, sono state analizzate sia in base alla tipologia di intervento descritta sia in relazione alla loro occorrenza in specifiche posizioni all'interno della struttura del testo.

\section{Caratteristiche linguistiche del corpus}

I testi analizzati presentano i caratteri peculiari del linguaggio medico ampiamente descritti in bibliografia (Serianni 2005). Oltre a un alto tasso di tecnicismi specifici e collaterali, e all'ampio uso di eponimi e acronimi, si attestano numerosi fenomeni di tipo morfologico e sintattico che risultano in alcuni casi chiari indizi di un'inclinazione alla brachilogia tipica del linguaggio medico e in particolare dei testi delle prassi. Tale tendenza risulta evidente nel frequente ricorso ad abbreviazioni ( $p . p$. per pneumoperitoneo, $a$. per arteria, $v$. per vena, ing. per inguinale, sec. per la preposizione secondo, $d x$, des e $d s$ per destro/a, sx, sin, sn per sinistro/a), nell'impiego di aggettivi di relazione giustapposti, in cui il primo aggettivo si presenta privo di suffisso (vescico-rettale, latero-terminale, epatoduodenale, gastro-duodenale, sieroemorragico, viscero-parietali) e nell'uso sostantivato dell'aggettivo per ellissi del nome (muscolo grande pettorale per fascia del muscolo grande pettorale, grande obliquo per muscolo grande obliquo, basilica per vena basilica, omerale per arteria omerale). Il fenomeno dell'ellissi si manifesta anche nella cancellazione di articoli determinativi, indeterminativi e partitivi (si reperta area pseudonodulare, si pone catetere vescicale, si evidenzia piccolo nodulo, si introduce ottica, si introduce rettoscopio operatorio, si evidenziano aderenze viscero-parietali, si apprezzano grossolani calcoli), e di preposizioni (nevo spalla, dito mano, capezzolo mammella).

Un'ulteriore caratteristica del linguaggio medico che si riscontra anche nel corpus è la propensione alla spersonalizzazione e alla cancellazione dell'agente, osservabile in particolare nell'uso esclusivo delle forme impersonali del verbo e della diatesi passiva ( $i$ reperti vengono inviati, $i$ vasi vengono sezionati, vengono asportati grossi linfonodi, la fascia del grande obliquo viene però ricostruita, il sacco viene isolato ed affondato). Questa tendenza si manifesta anche nella preferenza per la predicazione nominale in luogo di quella verbale. Nel corpus infatti si attesta un uso preponderante di frasi nominali $(72 \%)$. Se ne veda qualche esempio ai punti (1)-(4) ${ }^{8}$.

(1) posizionamento di rete in prolene sec. Lichtenstein

(2) incisione della linea alba

(3) asportazione di un lipoma di circa $5 \mathrm{~cm}$ di diametro

(4) isolamento e sezione tra clips metalliche degli elementi del peduncolo

Le frasi di tipo verbale invece presentano una frequenza nettamente inferiore $(28 \%)$ :

Per il rilevamento dei dati sottoposti ad analisi i testi sono stati preliminarmente processati in maniera automatica con il software NooJ, un ambiente di sviluppo linguistico per il Natural Language Processing con il quale è possibile creare dizionari elettronici e grammatiche sintattiche locali nella forma di automi a stati finiti (Silberztein 2015). I risultati estratti sono stati in seguito raffinati tramite controllo manuale. 
(5) si posiziona rete in prolene sec. Lichtenstein

(6) si incide la linea alba

(7) si asporta un lipoma di circa $10 \mathrm{~cm}$ di diametro

(8) si isolano e si sezionano tra clips metalliche gli elementi del peduncolo

La predicazione nominale, in quanto atemporale e non modale (cfr. infra), consente anche una rappresentazione astratta e generalizzabile dell'azione. Ciò ben si adatta all'espressione dell'attuazione di un protocollo e alla descrizione di una prassi chirurgica. Oltre alla deagentivizzazione, l'uso di costruzioni nominali risponde pure all'esigenza di condensazione tipica delle lingue speciali, e in particolare delle lingue tecnico-scientifiche (Cortelazzo 1994: 17-19). La tendenza all'economia si registra anche nei testi del corpus, che si caratterizzano infatti per una sintassi paratattica e priva di subordinazione in cui le azioni svolte dal medico sono espresse mediante il giustapporsi di frasi nominali di diverso tipo e, come si vedrà, principalmente di costruzioni nominali con nomi d'azioni'.

\section{Tipologie di frasi nominali nel corpus}

Secondo una definizione largamente condivisa, che ha come riferimento principale un noto saggio di Benveniste (1950 [1971]), la frase nominale rappresenta un enunciato assertivo finito in cui sussiste una normale relazione tra un soggetto e un predicato, al pari delle frasi verbali. La differenza è esclusivamente di natura morfologica, in relazione alla diversa classe, verbale o nominale, a cui è affidata la funzione predicativa. Il nome, diversamente dal verbo, non presenta determinazioni di tempo, persona e modo e pertanto la predicazione nominale «ha la caratteristica di essere atemporale, impersonale, non modale, in breve di poggiare su un termine ridotto al suo esclusivo contenuto semantico» (Benveniste 1950 [1971: 187]).

In italiano, la frase nominale presenta una gamma di possibilità di realizzazione molto vasta e, in parte, diamesicamente differenziata (cfr. Ferrari 2003) ${ }^{10}$. Le classificazioni proposte in bibliografia per lo scritto sono basate principalmente su testi letterari e giornalistici (Dardano 1986; Herczeg 1967; Mortara Garavelli 1971, 1973, 1974). Pur essendo l'uso di frasi nominali ampiamente diffuso in testi di ambito medico (Serianni 2005: 255), manca al momento una classificazione che dia conto in maniera sistematica dei differenti tipi di frase nominale che occorrono in questi testi e delle loro proprietà in relazione alla specifica tipologia testuale. Per quanto riguarda il corpus in esame, le frasi nominali individuate possono essere suddivise in tre tipi, come esemplificato di seguito ${ }^{11}$ :

$\left(a_{1}\right)$ colecisti distesa

$\left(a_{2}\right)$ non evidenti segni di flogosi

(b) presenza di voluminosa ernia

(c) induzione del pneumeperitoneo

Sull'uso dei nomi d'azione come strategia per costruire testi particolarmente sintetici si veda Fiorentino (2011). Sugli enunciati nominali nel parlato si vedano Cresti (1998), Ferrari (2003) e Scarano (2004).

11 Al fine di delineare una classificazione di portata più generale, ci si propone di verificare in futuro la presenza delle strutture qui individuate in corpora dello stesso tipo provenienti da altre strutture ospedaliere e in corpora di tipologia testuale affine (referti strumentali). 
Il tipo (a) è una struttura bimembre in cui il soggetto è rappresentato da un nome concreto mentre la funzione predicativa è affidata ad un aggettivo $\left(a_{1}\right)$ o ad una forma nominale del verbo, nello specifico ad un participio presente $\left(a_{2}\right)^{12}$.

I tipi (b) e (c), invece, sono rappresentati da costrutti nominali che contengono un nome derivato. Tale nome, frutto di una nominalizzazione morfologica, è inserito «in un SN che esprime il contenuto di una intera frase» (Castelli 1988: 333). Per nominalizzazione si intende il processo di transcategorizzazione in nome di elementi appartenenti a qualsiasi classe lessicale ${ }^{13}$. In italiano, tale processo si realizza attraverso mezzi morfologici o sintattici. La nominalizzazione morfologica forma nomi da verbi o aggettivi attraverso suffissi nominalizzatori o attraverso conversione (Castelli 1988; Grossmann / Rainer 2004: 293-381).

Nel tipo (b) la testa del SN, rappresentata nel corpus esclusivamente dai tipi lessicali assenza e presenza, è costituita da un nome di qualità morfologicamente derivato da un aggettivo in -nte mediante l'aggiunta del suffisso - (z)a (Grossmann / Rainer 2004: 293-381). L'argomento esterno del nome deaggettivale è introdotto dalla preposizione $d i$.

Nel tipo (c) la testa del SN è un nome morfologicamente derivato da un verbo transitivo. Si tratta di un nome d'azione, risultato di una nominalizzazione incentrata su un predicato d'azione, parafrasabile con una frase che contiene un verbo o con espressioni quali “l'azione di V, l'atto di V"14. I nomi d'azione attestati nel corpus sono formati principalmente con i suffissi -zione (introduzione, ricostruzione, asportazione, dissezione) e -mento (allestimento, ampliamento, posizionamento), e in misura minore con -ura (legatura, chiusura) o attraverso un processo di conversione (controllo, invio) (Grossmann / Rainer 2004: 323-338; 515-525).

In italiano, la struttura argomentale e la diatesi dei nomi d'azione si manifestano diversamente rispetto al verbo corrispondente ${ }^{15}$. In particolare, i nomi d'azione derivati da un verbo transitivo realizzano il Paziente come genitivo, mentre l'Agente è preferibilmente introdotto dalla locuzione passivizzante da parte $d^{16}$. Per quanto riguarda la diatesi, essa si manifesta non morfologicamente come nei verbi, ma sintatticamente nell'organizzazione della struttura argomentale. Tuttavia, non è sempre possibile stabilire la diatesi di tali costruzioni nominali laddove, come spesso accade, il nome d'azione presenta un unico argomento retto dalla preposizione $d i$. Ciò accade nei casi in cui il solo argomento espresso sia rappresentato da un nome

12 Nella classificazione proposta da Mortara Garavelli (1971) il tipo (a) è definito come frase «in cui sono riconoscibili, ben distinti, un soggetto e un predicato, nella reciproca relazione di portatore del messaggio e di attualizzatore» (Mortara Garavelli 1971: 278).

13 In bibliografia con nominalizzazione oltre che al processo di transcategorizzazione si fa riferimento al risultato di tale processo, ovvero al nome derivato (cfr. Koptjevskaja-Tamm 2006: 652). Per una definizione di nominalizzazione in prospettiva generale si vedano Chomsky (1970), Comrie (1976), Comrie / Thompson (1985) e Koptjevskaja-Tamm (1993, 2003). Per la nominalizzazione in italiano si veda Castelli (1988), Fiorentino (2004), Giorgi (1988) e Graffi (1994).

14 Herczeg (1967: 80) classifica le costruzioni nominali del tipo (c) quali «sostantivi astratti verbali in funzione indipendente».

15 Per un quadro complessivo delle strategie lessicali, sintattiche e semantiche a cui ricorrono le costruzioni nominali con nome d'azione per esprimere le proprietà verbali di persona, tempo, modo, aspetto e voce si veda Fiorentino (2004).

16 Non mancano tuttavia casi in cui entrambi gli argomenti sono realizzati come genitivi, dando luogo a costruzioni ambigue. L'ambiguità è risolta quando uno solo degli argomenti è animato oppure quando in base a fattori di lessico o a conoscenze enciclopediche risulti chiara l'assegnazione dei ruoli semantici (cfr. Fiorentino 2004: 25 n. 24; Giorgi 1988: 281-282). 
[+ animato]. Non sussistono invece ambiguità di questo tipo quando l'argomento espresso è un nome [- animato], che rende esplicita la diatesi passiva della costruzione. Le costruzioni del tipo (c) individuate nel corpus presentano sistematicamente un solo argomento [- animato] retto da di. Si tratta di costruzioni passive in cui non è espresso l'agente: una delle funzioni delle nominalizzazioni è proprio, come visto, la possibilità di cancellare l'agente o di riferirsi ad un agente generico (Ferrari 2002; Fiorentino 2004).

L'analisi si concentrerà sulle costruzioni nominali con nome d'azione (tipo c). Queste infatti sono altamente rappresentative della tipologia testuale oggetto di studio. I tipi (a) e (b) compaiono in modo limitato nel corpus, ed esclusivamente nelle sezioni del testo in cui sono riportate osservazioni sullo stato degli organi coinvolti o sulla patologia rilevata. Tali costruzioni sono invece tipiche di altre tipologie di testi delle prassi mediche, e in particolare dei referti strumentali.

\section{Costruzioni nominali con nome d'azione}

Le costruzioni nominali con nome d'azione rilevate nel corpus sono così rappresentabili:

$$
\mathrm{N}_{\text {azione }}+\text { Prep }+\mathrm{N}_{\text {concreto }}
$$

Rispetto a questa struttura di base, nel corpus compaiono alcune realizzazioni formali "alternative". In particolare, si attestano strutture nominali con argomento non introdotto da preposizione. Ne risulta un $\mathrm{SN}$ in cui sono giustapposti un nome testa a struttura argomentale, rappresentato dal nome d'azione, e un nome a struttura non argomentale, il nome concreto, che è il suo complemento:

$$
\mathrm{N}_{\text {azione }}+\mathrm{N}_{\text {concreto }}
$$

In tale struttura l'elemento posto a destra ha una funzione di specificazione restrittiva rispetto all'elemento posto a sinistra, come in posizionamento drenaggio ${ }^{17}$. Inoltre, ricorrono costruzioni ad un solo elemento nominale rappresentato da un nome concreto che risulterebbe essere l'argomento di una corrispondente struttura nominale con nome d'azione espresso (drenaggio in luogo di posizionamento di drenaggio):

$$
\mathrm{N}_{\text {concreto }}
$$

La tabella 1 riassume le frequenze di occorrenza delle diverse tipologie di strutture nominali finora osservate all'interno del corpus:

17 Giorgi (1988: 311-312) definisce i SN con argomenti non introdotti da preposizione, esemplificati da sollevamento pesi, rivendita tabacchi e da intestazioni e sigle come ufficio acquisti, costruzioni al limite tra struttura di sintagma e struttura di parola. 
Tabella 1. Frequenze dei tipi di strutture nominali nel corpus.

\begin{tabular}{|l|l|c|}
\hline \multicolumn{1}{|c|}{ Tipo } & \multicolumn{1}{c|}{ Esempio } & Frequenza \\
\hline $\mathrm{N}_{\text {azione }}+$ Prep $+\mathrm{N}_{\text {concreto }}$ & posizionamento di drenaggio & $85 \%$ \\
\hline $\mathrm{N}_{\text {azione }}+\mathrm{N}_{\text {concreto }}$ & posizionamento drenaggio & $6 \%$ \\
\hline $\mathrm{N}_{\text {concreto }}$ & drenaggio & $9 \%$ \\
\hline
\end{tabular}

Il tipo di struttura con argomento introdotto da preposizione, come posizionamento di drenaggio, controllo dell'emostasi, introduzione dell'ottica, costituisce quello maggiormente rappresentato, mentre le strutture "alternative" presentano percentuali d'occorrenza decisamente meno significative, sia per il tipo $\mathrm{N}_{\text {azione }}+\mathrm{N}_{\text {concreto }}$ sia per il tipo $\mathrm{N}_{\text {concreto }}$. Tuttavia, riguardo quest'ultimo, l'analisi distribuzionale operata sulla base dei tipi lessicali ha mostrato in relazione ad alcuni lessemi una frequenza superiore del tipo $\mathrm{N}_{\text {concreto }}$ rispetto a quella della corrispettiva costruzione con nome d'azione espresso ${ }^{18}$. Tale restrizione su base lessicale non si verifica invece per le strutture con giustapposizione di due elementi nominali. Le strutture del tipo $\mathrm{N}_{\text {azione }}+\mathrm{N}_{\text {concreto }}$ ricorrono in maniera sporadica all'interno del corpus e non mostrano carattere sistematico in nessun contesto esaminato:

Tabella 2. Frequenze di strutture $\mathrm{N}_{\text {azione }}+\mathrm{N}_{\text {concreto }}$ per tipi lessicali.

\begin{tabular}{|l|c|l|c|}
\hline \multicolumn{1}{|c|}{ Tipo lessicale } & Frequenza & \multicolumn{1}{c|}{ Tipo lessicale } & Frequenza \\
\hline Ricostruzione & $32,2 \%$ & Escissione & $2,8 \%$ \\
\hline Controllo & $29 \%$ & Asportazione & $1,9 \%$ \\
\hline Allestimento & $25 \%$ & Sutura & $1,5 \%$ \\
\hline Invio & $25 \%$ & Estrazione & $1,4 \%$ \\
\hline Ampliamento & $22,2 \%$ & Legatura & $1,1 \%$ \\
\hline Slittamento & $16,7 \%$ & Introduzione & $1 \%$ \\
\hline Elettrocauterizzazione & $12,5 \%$ & Resezione & $0,6 \%$ \\
\hline Incisione & $12,1 \%$ & Chiusura & $0,3 \%$ \\
\hline Dissezione & $5,2 \%$ & Induzione & $0,2 \%$ \\
\hline Rimozione & $3,3 \%$ & Posizionamento & $0,2 \%$ \\
\hline
\end{tabular}

In queste strutture 1'assenza della preposizione non configura sul piano sintattico un particolare tipo di costruzione nominale, ma rappresenta il risultato dell'ellissi della preposizione che introduce l'argomento del nome d'azione. Tale feno-

18 I tipi lessicali in questione sono riportati nella tabella 3. 
meno rientra nel più ampio quadro dei processi di ellissi grammaticale, tipici del linguaggio scientifico, che agiscono su determinanti e preposizioni ${ }^{19}$. All'interno del corpus l'ellissi della preposizione si riscontra sia nell'ambito delle costruzioni nominali con nome d'azione (controllo emostasi, ampliamento margini, asportazione lipoma), sia in SN in cui la preposizione ha la funzione di introdurre un elemento modificatore del nome (nevo alluce, collo piede). L'ellissi grammaticale rientra nella generale tendenza alla brachilogia che, come visto, costituisce un aspetto caratteristico dei testi delle prassi mediche. Il fenomeno tuttavia non risulta particolarmente pervasivo nel corpus e appare limitato ad alcuni casi sporadici. Le percentuali mostrano di fatto un quadro di generale tenuta delle preposizioni in questi contesti.

\subsection{Strutture ad un solo elemento con nome non argomentale}

I nomi concreti che costituiscono le strutture nominali ad un solo elemento indicano strumenti e dispositivi medico-chirurgici oppure parti del corpo. Di seguito si riportano le frequenze di occorrenza in relazione a ciascun tipo lessicale attestato:

Tabella 3. Frequenze di strutture $\mathrm{N}_{\text {concreto }}$ per tipi lessicali.

\begin{tabular}{|l|c|l|c|}
\hline \multicolumn{1}{|c|}{ Tipo lessicale } & Frequenza & \multicolumn{1}{c|}{ Tipo lessicale } & Frequenza \\
\hline Dufour & $100 \%$ & Catetere & $58,3 \%$ \\
\hline Strip & $100 \%$ & Cute & $61,3 \%$ \\
\hline Zaffo & $100 \%$ & Punti & $60,9 \%$ \\
\hline Redon & $95,8 \%$ & Plug & $54,8 \%$ \\
\hline Foley & $90 \%$ & Sottocute & $51,3 \%$ \\
\hline Drenaggio & $86,7 \%$ & Ottica & $18,8 \%$ \\
\hline Tampone & $83,3 \%$ & Piani & $6,7 \%$ \\
\hline Tubo & $79 \%$ & Parete & $0,2 \%$ \\
\hline Clips & $75 \%$ & Rete & $0,1 \%$ \\
\hline
\end{tabular}

Come si vede dalla tabella 3 , nella maggior parte dei casi le strutture di tipo Nconcreto hanno una frequenza di occorrenza maggiore di quelle in cui invece il nome d'azione è espresso. Dopo aver verificato la distribuzione lessicale, sono state prese in considerazione sia la specifica tipologia di intervento sia la posizione occupata dalle costruzioni nominali nella struttura del testo. Come si vedrà, sotto determinate condizioni, questi due fattori sembrano influire sull'emergere di tali costruzioni in quanto il nome d'azione omesso risulta recuperabile dal contesto. Di seguito saranno presentate alcune delle strutture ad un solo elemento individuate nel corpus.

19 Sull'ellissi grammaticale nel linguaggio scientifico si rimanda a Ignone (1991). 


\subsubsection{Il tipo catetere}

Le frequenze di occorrenza delle strutture ad un solo elemento nominale con il tipo catetere e l'eponimo Foley superano in modo significativo quelle delle strutture corrispondenti in cui è invece presente il nome d'azione (posizionamento, rimozione, sostituzione, invio). Nel caso dell'eponimo Dufour invece si attesta soltanto la forma ad un solo elemento.

Tabella 4. Frequenze delle strutture nominali con tipo lessicale catetere.

\begin{tabular}{|l|c|}
\hline \multicolumn{1}{|c|}{ Tipo } & Frequenza \\
\hline Catetere (sovrapubico, vescicale) & $58,3 \%$ \\
\hline Posizionamento di catetere & $16,7 \%$ \\
\hline Rimozione del catetere & $11,1 \%$ \\
\hline Rimozione CVC (catetere venoso centrale) & $5,6 \%$ \\
\hline Sostituzione di CVC & $2,8 \%$ \\
\hline Sostituzione di catetere & $2,8 \%$ \\
\hline Invio catetere & $2,8 \%$ \\
\hline
\end{tabular}

Tabella 5. Frequenze delle strutture nominali con l'eponimo Dufour.

\begin{tabular}{|c|c|}
\hline \multicolumn{1}{|c|}{ Tipo } & Frequenza \\
\hline Dufour & $100 \%$ \\
\hline
\end{tabular}

Tabella 6. Frequenze delle strutture nominali con l'eponimo Foley.

\begin{tabular}{|l|c|}
\hline \multicolumn{1}{|c|}{ Tipo } & Frequenza \\
\hline Foley & $90 \%$ \\
\hline Posizionamento di Foley & $10 \%$ \\
\hline
\end{tabular}

Le strutture catetere, Foley e Doufur ricorrono in luogo della frase nominale posizionamento di catetere, posizionamento di Foley, posizionamento di Dufour. Queste forme risultano frequenti in specifici ambiti di occorrenza, in particolare nelle descrizioni di interventi chirurgici di tipo urologico, come la resezione transuretrale della vescica (TURV) e della prostata (TURP). In questi interventi il posizionamento di catetere è previsto dalla prassi e pertanto le relative descrizioni si caratterizzano per la presenza di una specifica sezione informativa associata a questa azione chirurgica: 
(9) Agevole introduzione del resettore $26 \mathrm{ch}$ previa uretrotomia con Otis fino a $28 \mathrm{ch}$. Mucosa vescicale sana, meati in sede, collo sollevato da I.P.B. Uretra indenne. TURP previa epicistostomia con cannula di Reuter. Catetere sovrapubico $14 \mathrm{ch}$, Catetere vescicale $20 \mathrm{ch}$.

(10) Introduzione dello strumento in vescica. Pareti vescicali regolari. Meati ureterali in sede. In sede perimeatale sinistra presenza di neoformazione sessile, di circa $1,5 \mathrm{~cm}$, rivestita da epitelio macroscopicamente normale e di aspetto solido al taglio. Si procede a TURV con prelievo profondo. Emostasi. Catetere vescicale a tre vie e lavaggio urologico.

(11) Posizionato il resettore ch 24, si procede a duplice incisione del collo vescicale e si asporta il tessuto sclerotico; si passa quindi a resecare la prostata con taglio lungo per quadranti successivi; accurata emostasi della loggia prostatica e Dufour ch 24 in vescica per cistoclisi continua.

Le occorrenze di rimozione del catetere, sostituzione del catetere e invio catetere rappresentano nel corpus dei casi marcati in quanto la rimozione, la sostituzione o l'invio del catetere non figurano nelle normali pratiche descritte negli interventi urologici. Queste attività sono di solito praticate nei giorni successivi all'operazione chirurgica e quindi non rientrano nella relazione descrittiva dell'intervento urologico. Nel corpus rimozione del catetere e sostituzione del catetere si ritrovano infatti soltanto in testi relativi ad interventi non urologici (come in 12), oppure in quelli in cui l'intervento ha il solo scopo di sostituire il catetere (come in 13,14 e 15):

(12) Induzione del pneumo peritoneo con ago di Veress, si introducono gli altri trocars ai soliti punti di repere con trocar da $10 \mathrm{~mm}$. all'ombelico [...] $R i$ mozione del catetere chiusura tra clips del cistico e preparazione e sezione tra clips dell'arteria cistica. Colecistectomia VLS, controllo dell'emostasi, toilette del cavo, drenaggio tubulare sottoepatico, sutura delle mini-incisioni.

(13) Incisione ellittica intorno all'uscita cutanea del catetere, scollamento dell'anello in Dacron lisi della pellicola fibrotica intorno al catetere. Rimozione del catetere e sutura a strati della incisione.

(14) Previa rimozione CVC perché trombizzato, viene impiantato nuovo CVC in giugulare destra. Controllo della funzionalità.

(15) Sostituzione del catetere vescico-uretrale ch. 22. Preparazione del tratto uretrale interessato e sua ricostruzione in continua con lembo a cerniera mediana. Preparazione dei lembi cutanei e copertura dell'uretra.

In questi casi non si attestano le strutture catetere, Foley o Dufour in quanto i nomi d'azione rimozione e sostituzione non sono facilmente recuperabili dal contesto: nei testi come in (12) in quanto l'azione chirurgica denotata da rimozione non è prevista dalla prassi dell'intervento di colecistectomia descritto; in (13), (14) e (15) perché il tipo di intervento è scarsamente documentato e dunque le relative descrizioni non presentano una regolarità di struttura tale che l'occorrenza in una determinata sezione informativa possa rendere recuperabile il nome d'azione. 


\subsubsection{Il tipo cute}

Anche con il tipo lessicale cute, la struttura ad un solo elemento occorre con maggiore frequenza rispetto alle realizzazioni in cui cute costituisce l'argomento di un nome d'azione, sia introdotto da preposizione sia con cancellazione della preposizione.

Di seguito sono riportate le frequenze di occorrenza di ciascun tipo attestato:

Tabella 7. Frequenze delle strutture nominali con il tipo lessicale cute.

\begin{tabular}{|l|c|}
\hline \multicolumn{1}{|c|}{ Tipo } & Frequenza \\
\hline Cute & $61,3 \%$ \\
\hline Sutura della cute & $28,2 \%$ \\
\hline Sutura cute & $5,1 \%$ \\
\hline Incisione della cute & $2,8 \%$ \\
\hline Ricostruzione della cute & $1,4 \%$ \\
\hline Innesto di cute & $0,6 \%$ \\
\hline Ricostruzione cute & $0,3 \%$ \\
\hline Rimodellamento della cute & $0,3 \%$ \\
\hline
\end{tabular}

Le strutture ad un solo elemento si ritrovano esclusivamente nelle sezioni finali delle descrizioni degli interventi. Poiché tali sezioni sono solitamente dedicate alle azioni chirurgiche relative alla "sutura", è possibile considerare il tipo cute come corrispondente a sutura della cute ${ }^{20}$. Le azioni chirurgiche indicate dai nomi d'azione incisione, innesto e rimodellamento, infatti, costituiscono attività differenti rispetto alla sutura e pertanto non ricorrono mai nelle sezioni finali dei testi. I tipi sutura della cute, sutura cute e cute ricorrono invece nel medesimo contesto di occorrenza:

(16) Incisione sull'emiscroto sn., svuotamento esteriorizzazione della gonade, eversione e resezione della vaginale, drenaggio laminare, controllo dell'emostasi, sutura della cute.

(17) Incisione a losanga escidente in blocco la fistola e i suoi tragitti fistolosi. Controllo dell'emostasi. Tubo di drenaggio e sutura cute.

(18) Incisione QIE/QII mammella sinistra. Scollati il piano cutaneo e preghiandolare si reperta area di aumentata consistenza che viene ampiamente asportata. Emostasi accurata, drenaggio laminare, ricostruzione corpus mammae. Cute a punti staccati.

20 Un caso problematico è rappresentato dalle strutture in cui ricorre il tipo lessicale ricostruzione. Sebbene, in generale, i termini sutura e ricostruzione non possano considerarsi sinonimici, l'analisi mostra che il termine ricostruzione in associazione a cute ricorre nel corpus in contesti sovrapponibili a quelli di sutura della cute. Pertanto l'interpretazione proposta di cute come sutura della cute a nostro avviso resta valida, anche in considerazione della bassissima frequenza delle strutture ricostruzione della cute e ricostruzione cute. 
Dall'analisi degli specifici contesti di questi tipi è emersa una distribuzione non omogenea rispetto alla tipologia di intervento. Si attesta infatti una maggiore concentrazione delle forme cute nell'ambito delle descrizioni degli interventi di chirurgia della mammella (mastectomie, ricostruzioni, quadrantectomie):

(19) Incisione QIE/QII mammella sinistra. Scollati il piano cutaneo e preghiandolare si reperta area di aumentata consistenza che viene ampiamente asportata. Emostasi accurata, drenaggio laminare, ricostruzione corpus mammae. Cute a punti staccati.

(20) Incisione periareolare QII/QIE della mammella di sinistra. Scollato il piano cutaneo e pre-ghiandolare, si reperta un nodulo polilobato con diametro maggiore di circa $2 \mathrm{~cm}$ che viene asportato. Emostasi, ricostruzione del corpus. Cute in intradermica.

(21) Incisione arcuata periareolare QSE/QSI sul reperage cutaneo ecoguidato; scollato il piano cutaneo e pre-ghiandolare si reperta area di consistenza duro-fibrotica. Si procede ad ampia escissione sino a sotto il capezzolo. Emostasi accurata, posizionamento di drenaggio in aspirazione. Ricostruzione corpus mammae, cute a punti staccati.

(22) Incisione sul QSE di sn; scollato il piano cutaneo e pre-ghiandolare si reperta nodulo di diametro $2 \mathrm{~cm}$ che viene asportato. Emostasi accurata, ricostruzione corpus mammae; cute in intradermica.

Negli interventi di chirurgia della mammella la sutura è solitamente rappresentata da una singola azione chirurgica, ovvero la sutura della cute. In altri interventi, invece, in cui pure compare la sutura della cute, l'intero tempo chirurgico dedicato alla sutura può essere articolato in diverse fasi, a ciascuna delle quali corrisponde una specifica sezione informativa nella struttura testuale dell'intervento:

(23) Riperitoneizzazione del cavo pelvico, confezionamento di ano iliaco definitivo sutura della parete e della cute a strati.

(24) Posizionamento di rete in polipropilene sec. Lichtenstein, sutura a strati della parete e della cute.

(25) Incisione inguinale destra. Presenza di ernia diretta che viene isolata ed affondata. Grosso lipoma del canale inguinale che viene resecato. Rinforzo della fascia trasversale e plastica sec. Lichtenstein. Sutura a strati e sutura della cute.

L'emergere di forme ad un solo elemento nominale cute nei testi relativi agli interventi alla mammella sembra favorito dall'alto grado di prevedibilità dell'azione chirurgica "sutura della cute" in quanto unica tipologia di sutura riscontrabile nelle descrizioni di questi interventi. In tali casi si rileva il solo nome non argomentale, l'elemento con maggiore carico informativo, mentre l'informazione "sutura" è già data dalla posizione occupata dal tipo cute all'interno della struttura del testo.

\subsubsection{Il tipo plug}

Nel corpus ricorrono due tipi di strutture con nome concreto plug: la forma ad un solo elemento plug, solitamente accompagnata dall'aggettivo anale, e la struttura in cui il 
nome concreto, introdotto dalla preposizione $d i$, è l'argomento di un nome d'azione. La struttura ad un solo elemento rappresenta quella con frequenze maggiori:

Tabella 8. Frequenze delle strutture nominali con il tipo lessicale plug.

\begin{tabular}{|l|c|}
\hline \multicolumn{1}{|c|}{ Tipo } & Frequenza \\
\hline Plug (anale) & $54,8 \%$ \\
\hline Posizionamento di plug & $41,1 \%$ \\
\hline Apposizione di plug & $1,4 \%$ \\
\hline Applicazione di plug & $1,4 \%$ \\
\hline Rimozione del plug & $1,4 \%$ \\
\hline
\end{tabular}

Dall'analisi dei contesti emerge che il tipo plug ricorre esclusivamente nelle descrizioni degli interventi di chirurgia proctologica, come fistulotomie ed emorroidectomie. In queste tipologie di intervento, infatti, l'applicazione di un plug anale è prevista dalla prassi chirurgica, e occupa una specifica sezione nella struttura del testo:

(26) Previa divaricazione con anoscopio operatorio si reperta posteriormente profonda ragade anale con orifizio interno che comunica con due tramiti fistolosi intersfinterici. Si esegue fistulotomia dei tramiti. Apertura del piano intersfinterico con curettage dell'ascesso cronico. Controllo dell'emostasi. Plug anale.

(27) Previa divaricazione con anoscopio operatorio si repertano tre grossi gavoccioli emorroidali a ore 3-6-11. Si esegue dearterizzazione dei rami emorroidali. Emorroidectomia con Ligasure. Controllo dell'emostasi. Plug anale.

(28) Previa divaricazione con anoscopio operatorio si repertano due gavoccioli emorroidari. Si esegue emorroidectomia con ligasure. Controllo dell'emostasi, plug anale.

Le strutture con nome d'azione espresso si ritrovano in modo quasi esclusivo in interventi differenti da quelli proctologici. In questi casi sono adoperati plug di tipo diverso da quello anale e il loro impiego non è sempre previsto dalla prassi chirurgica, ma può essere richiesto dalle specifiche esigenze di un dato caso clinico:

(29) Incisione inguinale destra. Dissociati i piani superficiali si individua un sacco erniario che impegna l'anello crurale. Preparazione e riduzione e posizionamento di plug. Plastica del canale inguinale.

(30) Incisione verticale sulla tumefazione, si reperta un sacco da ernia crurale destra che viene isolato, resecato ed affondato. Posizionamento di plug in polipropilene.

Di nuovo, la presenza della struttura ad un solo elemento sembrerebbe dipendere dalla recuperabilità del nome d'azione omesso sulla base del contesto e, nello specifico, della tipologia di intervento descritto nel verbale. 


\subsubsection{Il tipo drenaggio}

Il tipo drenaggio rappresenta un ulteriore esempio in cui la struttura ad un solo elemento nominale ricorre con maggiore frequenza del corrispettivo tipo con nome d'azione espresso. Oltre alla forma drenaggio, si attestano nel corpus anche la forma eponimica Redon e il nome concreto tubo accompagnato da un SP di drenaggio o di Redon.

Tabella 9. Frequenze delle strutture nominali con il tipo lessicale drenaggio.

\begin{tabular}{|l|c|}
\hline \multicolumn{1}{|c|}{ Tipo } & Frequenza \\
\hline Drenaggio & $86,7 \%$ \\
\hline Posizionamento di drenaggio & $12,3 \%$ \\
\hline Posizionamento drenaggio & $0,6 \%$ \\
\hline Asportazione drenaggio & $0,1 \%$ \\
\hline Inserimento di drenaggio & $0,1 \%$ \\
\hline Inserzione di drenaggio & $0,1 \%$ \\
\hline Riposizionamento di drenaggio & $0,1 \%$ \\
\hline
\end{tabular}

Tabella 10. Frequenze delle strutture nominali con il tipo lessicale tubo.

\begin{tabular}{|l|c|}
\hline \multicolumn{1}{|c|}{ Tipo } & Frequenza \\
\hline Tubo (di drenaggio, di Redon) & $79 \%$ \\
\hline Posizionamento di tubo & $21 \%$ \\
\hline
\end{tabular}

Tabella 11. Frequenze delle strutture nominali con il tipo lessicale Redon.

\begin{tabular}{|l|c|}
\hline \multicolumn{1}{|c|}{ Tipo } & Frequenza \\
\hline Redon & $95,8 \%$ \\
\hline Posizionamento di Redon & $4,2 \%$ \\
\hline
\end{tabular}

Le forme nominali drenaggio, tubo di drenaggio, tubo di Redon, Redon ricorrono nel medesimo contesto di occorrenza di posizionamento di drenaggio, rappresentato nella struttura del testo dalla sezione informativa "posizionamento di drenaggio":

(31) Induzione del pneumoperitoneo con ago di Veress, si introducono i trocars ai soliti punti di repere con $\mathrm{mm}$. all'ombelico [...] Lisi delle aderenze, preparazione e sezione tra clips degli elementi del peduncolo, colecistectomia VLS e sua estrazione in endobag. Controllo dell'emostasi, toilette del cavo, posizionamento di drenaggio sottoepatico, sutura delle incisioni. 
(32) Incisione scrotale a sin. Esteriorizzazione della gonade. Presenza di vasi ectasici e di liquido intravaginale. Legatura di alcuni vasi ectasici ed eversione della vaginale. Controllo emostasi, drenaggio laminare, sutura della incisione.

(33) Taglio a cravatta di Kocher, divaricati i muscoli pretiroidei si apprezza una formazione nodulare che occupa tutto il lobo di ds. Lobo sn. normoconformato. Si procede a legatura dei peduncoli vascolari e tiroidectomia sub totale. Controllo dell'emostasi, tubo di drenaggio in aspirazione, sutura a strati della parete.

(34) Incisione a losanga includente alcuni tramiti fistolosi. Fistolectomia in blocco fino alla fascia presacrale. Controllo emostasi. Redon in aspirazione. Sutura a strati dell'incisione.

Come possiamo notare dagli esempi, differentemente dai casi di catetere e cute, le strutture in cui ricorre il tipo drenaggio presentano una distribuzione trasversale e non caratteristica di un particolare intervento. Infatti, l'applicazione di un drenaggio è una pratica comune a varie tipologie di operazione chirurgica e pertanto la sezione ad essa associata ricorre nella struttura testuale delle relazioni di diversi interventi. In questo caso è l'alta frequenza dell'attività nella prassi chirurgica a favorire l'ellissi del nome d'azione e dunque la realizzazione di strutture ad un solo elemento nominale.

\section{Conclusioni}

Dall'analisi condotta emerge che nel corpus in esame i tipi "alternativi" alla struttura nominale $\mathrm{N}_{\text {azione }}+$ Prep $+\mathrm{N}_{\text {concreto }}$ sono il risultato di due differenti processi di ellissi. $\mathrm{Nel}$ caso di $\mathrm{N}_{\text {azione }}+\mathrm{N}_{\text {concreto }}$ agisce sulla struttura l'ellissi grammaticale che si manifesta nella cancellazione della preposizione. Tale fenomeno, come visto, dipende da esigenze di economia volte a eliminare elementi privi di un contenuto semantico proprio che si riscontra non solo nelle costruzioni nominali con nome d'azione, ma, in modo trasversale, anche quando la preposizione instaura rapporti sintattici di tipo differente, ad esempio come elemento introduttore di un modificatore del nome.

Le strutture $\mathrm{N}_{\text {concreto }}$ sono invece l'esito di un processo di ellissi contestuale in cui l'elemento omesso è il nome d'azione. La recuperabilità di tale elemento dipende da fattori di diverso genere. In primo luogo assume un peso determinante l'alta specificità dell'azione chirurgica espressa dalla costruzione nominale in relazione a una particolare tipologia di intervento. Esemplificativi in tal senso sono i casi di catetere e plug. Si è visto infatti che le due strutture compaiono soltanto nelle descrizioni di interventi in cui il posizionamento del dispositivo costituisce un'azione fondamentale e caratterizzante della prassi operatoria. In altri casi invece, come visto con il tipo cute, il nome d'azione risulta recuperabile sulla base della posizione occupata dall'elemento all'interno della struttura della descrizione di un dato intervento. In ultimo, un'altra condizione per la recuperabilità del nome d'azione è rappresentata dalla frequenza di una data operazione non solo nella prassi chirurgica di un singolo ma di una pluralità di interventi. Questa casistica è stata illustrata dal tipo drenaggio.

Entrambi i processi di ellissi, sebbene di natura diversa, sono espressione di una tendenza brachilogica e rispondono quindi all'esigenza di brevità e sinteticità pro- 
pria del linguaggio medico e, nel caso specifico dei verbali chirurgici, al bisogno pratico di velocizzare la scrittura. La ripetitività delle prassi operatorie, codificata nei testi in una sequenza di frasi che ricorrono in forma identica, permette la soppressione di elementi resi altamente prevedibili dal contesto per chi condivide le medesime conoscenze dello scrivente. Laddove si devia da tale prassi gli operatori sono invece costretti ad un'esposizione maggiormente precisa e dettagliata.

\section{Riferimenti bibliografici}

Benveniste, Émile (1950 [1971]): «La phrase nominale», Bulletin de la Société de linguistique de Paris, 46, pp. 19-36. [Tr. it. «La frase nominale», in É. Benveniste, Problemi di linguistica generale, Milano, Il Saggiatore, pp. 179-199].

Castelli, Margherita (1988): «La nominalizzazione», in L. Renzi / G. Salvi / A. Cardinaletti (a c. di), Grande grammatica italiana di consultazione, Vol. I, Bologna, Il Mulino, pp. 332-356.

Chomsky, Noam (1970): «Remarks on nominalization», in R. A. Iacobs / P. S. Rosenbaum (eds.), Readings in English Transformational Grammar, Waltham (MA), Ginn, pp. 184-221.

Comrie, Bernard (1976): «The syntax of action nominals: a cross-language study», Lingua, 40, pp. $177-201$.

Comrie, Bernard / Thompson, Sandy (1985): «Lexical nominalization», in T. Shopen (ed.), Language Typology and Syntactic Description, Vol. 3, Cambridge, Cambridge University Press, pp. 349-398.

Cortelazzo, Michele (1994): Lingue speciali. La dimensione verticale, Padova, Unipress.

Cresti, Emanuela (1998): «Gli enunciati nominali», in M. T. Navarro Salazar (ed.), Italica matritensia. Atti del IV Congresso della Società Internazionale di Linguistica e Filologia Italiana (Madrid, 27-29 giugno 1996), Firenze, Cesati, pp. 171-191.

Dardano, Maurizio (1986): Il linguaggio dei giornali italiani, Bari, Laterza.

Ferrari, Angela (2002): «Aspetti semantici e informativi della nominalizzazione sintagmatica», in G. L. Beccaria / C. Marello (a c. di), La parola al testo. Scritti per Bice Mortara Garavelli, Alessandria, Edizioni dell'Orso, pp. 179-204.

Ferrari, Angela (2003): «Le frasi nominali nel parlato e nello scritto», in E. Burr (a c. di), Tradizione \& innovazione. Linguistica e filologia italiana alle soglie di un nuovo millennio. Atti del VI Convegno SILFI (Duisburg, 28 giugno-2 luglio 2000), Firenze, Cesati, pp. 170-189.

Fiorentino, Giuliana (2004): «Nomi d'azione e subordinazione in italiano», Studi e Saggi Linguistici, 42, pp. 9-41.

Fiorentino, Giuliana (2011): «Action nouns between speech and writing: The case of Italian», Rivista di Linguistica, 23 (1), pp. 39-57.

Giorgi, Alessandra (1988): «La struttura interna dei sintagmi nominali», in L. Renzi / G. Salvi / A. Cardinaletti (a c. di), Grande Grammatica Italiana di Consultazione, vol. 1, Bologna, Il Mulino, pp. 273-314.

Graffi, Giorgio (1994): Sintassi, Bologna, Il Mulino.

Grossmann, Maria / Rainer, Franz (a c. di) (2004): La formazione delle parole in italiano, Tübingen, Niemeyer.

Herczeg, Giulio (1967): Lo stile nominale in italiano, Firenze, Le Monnier.

Ignone, Anna (1991): «L'ellissi nel linguaggio scientifico», Studi italiani di linguistica teorica ed applicata, XX (3), pp. 519-540. 
Koptjevskaja-Tamm, Maria (1993): Nominalizations, London / New York, Routledge.

Koptjevskaja-Tamm, Maria (2003): «Action nominal constructions in the languages of Europe», in F. Plank (ed.), Noun Phrase Structure in the Languages of Europe, Berlin / New York, Mouton De Gruyter, pp. 723-759.

Koptjevskaja-Tamm, Maria (2006): «Nominalization», in K. Brown (ed), Encyclopedia of Language and Linguistics., Vol. 8, Amsterdam, Elsevier, 2nd edition, pp. 652-659.

Kremer, Karl et alii (a c. di) (1990-1999): Grande atlante di tecnica chirurgica, 11 voll., Firenze, USES.

Mortara Garavelli, Bice (1971): «Fra norma e invenzione: lo stile nominale», Studi di grammatica italiana, 1, pp. 271-315.

Mortara Garavelli, Bice (1973): «Lineamenti di una tipologia dello stile nominale nella prosa letteraria contemporanea», in M. Gnerre / M. Medici / R. Simone (a c. di), Storia linguistica dell'Italia del Novecento, Roma, Bulzoni, pp. 113-125.

Mortara Garavelli, Bice (1974): «Lo stile nominale nella lingua giornalistica: proposte per un'analisi testuale», in AA.VV., Italiano d'oggi. Lingua non letteraria e lingue speciali, Trieste, LINT, pp. 227-236.

Paletto, Angelo Emilio (a c. di) (1996-2006): Nuovo trattato di tecnica chirurgica, 6 voll., Torino, UTET.

Scarano, Antonietta (2004): «Enunciati nominali in un corpus di italiano parlato. Appunti per una grammatica corpus based», in F. Albano Leoni / F. Cutugno / M. Pettorino / R. Savy (a c. di), Il parlato italiano. Atti del Convegno nazionale (Napoli, 13-15 febbraio 2003), Napoli, M. D’Auria, pp. 1-18.

Serianni, Luca (1989): Saggi di storia linguistica italiana, Napoli, Morano Editore.

Serianni, Luca (2003): Italiani scritti, Bologna, Il Mulino.

Serianni, Luca (2005): Un treno di sintomi. I medici e le parole: percorsi linguistici nel passato e nel presente, Milano, Garzanti.

Silberztein, Max (2015): La formalisation des langues. L'approche de NooJ, London, ISTE Éditions.

Vella, Luciano (a c. di) (2008): Enciclopedia medica italiana, Torino, USES. 